\title{
TDRG I functions in testicular seminoma are dependent on the PI3K/Akt/mTOR signaling pathway
}

\author{
Yong Wang' \\ Yu Gan' \\ Zhengyu Tan' \\ Jun Zhou' \\ Riko Kitazawa ${ }^{2}$ \\ Xianzhen Jiang' \\ Yuxin Tang' \\ Jianfu Yang'
}

'Department of Urology, The Third Xiangya Hospital of Central South University, Changsha, People's Republic of China; ${ }^{2}$ Department of Diagnostic Pathology, Ehime University Hospital, Shitsukawa, Tōon, Ehime Perfecture, Japan
Correspondence: Yuxin Tang; Jianfu Yang Department of Urology, The Third Xiangya Hospital of Central South University, 138 Tong Zipo Road, Changsha 4I0013, People's Republic of China Email mmcct@।26.com;

jeffxy3yy@।63.com
This article was published in the following Dove Press journal:

OncoTargets and Therapy

20 January 2016

Number of times this article has been viewed
Abstract: Human testis development-related gene 1 (TDRG1) is a recently identified gene that is expressed exclusively in the testes and promotes the development of testicular germ cell tumors. In this study, the role of TDRG1 in the development of testicular seminoma, which is the most common testicular germ cell tumor, was further investigated. Based on polymerase chain reaction, Western blotting, and immunohistochemistry tests, both gene and protein expression levels of TDRG1 were significantly upregulated in testicular seminoma tissues compared with normal testicular tissues. Additionally, the levels of phosphoinositide-3 kinase (PI3K)/p1 10 and Akt phosphorylation were dramatically upregulated in testicular seminoma tissues. Accordingly, in our cell experiment, seminoma TCam-2 cells were subjected to different treatments: the TDRG1 knockout, TDRG1 overexpression, PI3K inhibition (LY294002 administration), or PI3K activation (insulin-like growth factor-1 administration). Cell proliferation, the proliferation index, apoptosis rate, cell adhesive capacity, and cell invasion capability were assessed. Cells with both TDRG1 knockout and PI3K inhibition exhibited decreased cell proliferation, proliferation indexes, cell adhesion capacity, and cell invasion capability and increased apoptosis rates. Most of these effects were reversed by TDRG1 overexpression or PI3K activation, indicating that both $T D R G 1$ - and PI3K-mediated signaling promote proliferation and invasion of testicular seminoma cells. The knockout of TDRG1 significantly decreased the phosphorylation levels of $\mathrm{PI} 3 \mathrm{~K} / \mathrm{p} 85$, PI3K/p110, Akt, and mammalian target of rapamycin (mTOR; Ser ${ }^{2448}$ ). Except for $\mathrm{PI} 3 \mathrm{~K} / \mathrm{p} 110, T D R G 1$ overexpression had the opposite effects on phosphorylation levels. Phosphorylated mTOR at $\mathrm{Ser}^{2481}$ and $\mathrm{Thr}^{2446}$ was not affected by TDRG1 or PI3K in our tests. Thus, these results indicate that TDRG1 promotes the development and migration of seminoma cells via the regulation of the PI3K/Akt/mTOR signaling pathway; this contributes to an understanding of the precise mechanisms underlying the development and migration of seminomas and lays a theoretical foundation for the development of appropriate therapies.

Keywords: TDRG1, testicular seminoma, PI3K, Akt, mTOR

\section{Introduction}

Testicular seminoma accounts for $40 \%-60 \%$ of all testicular germ cell tumors, which are the most common solid tumors in males aged $15-35$ years. ${ }^{1,2}$ Although the cure rate of testicular seminoma at stage I (nonmetastasized seminoma) exceeds 95\%, there is a poor prognosis once the tumor spreads to adjacent lymph node groups (including those in the neck, mediastinum, and abdomen), lung, liver, and bones. ${ }^{2-6}$ Epidemiological studies have indicated that cryptorchidism, gonadal dysgenesis, familial testis cancer, and environmental exposure to chemical substances increase the risk of seminoma occurrence; however, the precise molecular pathogenesis of seminoma is unclear. ${ }^{2,7,8}$ In our previous studies, we identified human testis development-related gene 1 (TDRG1), 
which is expressed exclusively in the testes. It encodes a 100-amino acid protein that lacks characterized protein domains. ${ }^{9}$ We also found that inhibiting TDRG1 expression via RNA interference dramatically attenuates the ability of testicular germ cell tumor NTERA-2 cells to proliferate and invade. ${ }^{10}$ Accordingly, we speculated that TDRG1 acts as an oncogene in the testes.

The phosphoinositide-3 kinase (PI3K)/Akt signaling pathway plays a crucial role in tumorigenesis and tumor progression. Constitutive activation of PI3K/Akt signaling due to a variety of genetic aberrations is frequently observed in a wide spectrum of human cancers. ${ }^{11-13}$ Activated PI3K/Akt signaling is the central effector of many downstream signaling pathways that regulate various cellular responses of tumors, including cell growth, transformation, differentiation, motility, survival, and chemosensitivity. ${ }^{14,15} \mathrm{PI} 3 \mathrm{~K} / \mathrm{Akt}$ signaling is involved in the inhibition of apoptosis via the phosphorylation of proapoptotic molecules, such as p53, Bad, and caspase-9, or modulating transcription factors (eg, c-Raf). ${ }^{15}$ Additionally, the PI3K/Akt pathway that regulates NOS and GSK3 $\beta$ is required to control cell migration and angiogenesis. ${ }^{16,17}$ There are also reports that the PI3K/Akt signaling pathway is involved in breast, lung, gastric, and prostate cancers that become refractory to cytotoxic therapy. ${ }^{18}$

Mammalian target of rapamycin (mTOR), a serine/ threonine kinase, is downstream of PI3K/Akt and positively regulates tumor progression. ${ }^{19}$ Interest in the mTOR-mediated pathway was initially derived from the discovery of its specific inhibitor, rapamycin, an anticancer drug that has been used clinically. However, rapamycin has since been found to induce tumorigenesis in some cases, presumably because mTOR itself negatively regulates the PI3K/ Akt pathway. ${ }^{19}$ mTOR involvement in impeding tumor progression is closely related to the downstream effector molecule ribosomal protein S6 kinase (S6K1) and eukaryotic translation initiation factor 4E-binding protein 1 (4E-BP1). These molecules stimulate ribosome biogenesis to meet the increased demand for protein synthesis caused by tumor cell cycle progression. ${ }^{20}$ Additionally, mTOR is involved in the regulation of cyclin $\mathrm{D} 1$, cyclin-dependent kinases, c-myc, vascular endothelial growth factor, PKC, and NF- $\mathrm{BB}$, conferring advantages to cancer cells with respect to growth, transformation, differentiation, motility, and survival. ${ }^{21}$ Accordingly, PI3K/Akt and mTOR are candidate signaling pathways that may mediate the effect of TDRG1 in testicular germ cell tumors.

In the present study, the expression of TDRG1 at both the gene and the protein levels was examined to determine its role in testicular seminoma. We found that TDRG1 expression is remarkably higher in testicular seminoma tissues than in normal testicular tissues, indicating a positive role of the gene in the development and progression of testicular seminoma. Based on our cellular experiments, we provide clear evidence that TDRG1 promotes the proliferation and invasion of testicular seminoma cells. Our results are consistent with accumulating evidence supporting the relationship between aberrant activation of the PI3K/Akt/mTOR cascade and cell proliferation and invasion in many human cancers. The results of the present study will contribute to our understanding of the precise mechanisms underlying the proliferation and invasion of seminoma and lay a theoretical foundation for its therapy.

\section{Materials and methods Ethics statement}

This study was approved by the Ethics Committee of Xiangya School of Medicine (Changsha, People's Republic of China). All patients provided written informed consent in compliance with the code of ethics of the World Medical Association (Declaration of Helsinki; Ferney-Voltaire, France).

\section{Tissues, cells, and reagents}

Thirty tissue specimens from testicular seminoma tissues $(n=33)$ and normal testicular tissues $(n=33)$ were obtained from the Third Xiangya Hospital of Central South University and the Affliated Cancer Hospital of Xiangya School of Medicine. Polymerase chain reaction (PCR) and Western blotting were performed to detect the expression levels of TDRG1 in both tissue types. Testicular seminoma TCam-2 cells were obtained from Professor Riko Kitazawa (Department of Diagnostic Pathology, Ehime University Hospital, Matsuyama, Japan) and were cultured in RPMI 1640 medium at $37^{\circ} \mathrm{C}$ in a humidified $5 \% \mathrm{CO}_{2}$ atmosphere. Antibodies against PI3K/p85 (1:8,000), phospho-PI3K/p85 (p-PI3K/p85, 1:8,000), p-Akt (1:4,000), and p-mTOR (T2446, 1:4,000) were obtained from Abcam Biotechnology (Cambridge, UK); mTOR (1:4,000), p-mTOR (Ser2448, 1:4,000), and p-mTOR (Ser2481, 1:4,000) antibodies were purchased from Cell Signaling Technology (Boston, MA, USA); PI3K/ p1 $10(1: 4,000)$ and p-PI3K/p1 $10(1: 4,000)$ antibodies were obtained from Boaoshen Biotechnology (Beijing, People's Republic of China). The antibodies against TDRG1 $(1: 4,000)$ and glyceraldehyde-3-phosphate dehydrogenase (GAPDH) $(1: 8,000)$ were purchased from Acris Biotechnology (San Diego, CA, USA) and Santa Cruz Biotechnology Inc. (Dallas, TX, USA). LY294002 and insulin-like growth factor-1 (IGF-1), an inhibitor and activator of the PI3K pathway, respectively, were purchased from Selleck Chemicals (Houston, TX, USA). 


\section{Cell transfection}

An open-reading frame clone of Homo sapiens TDRG1 was subcloned into the enhanced green fluorescent protein plasmid-C1 (pEGFP-C1) vector (Thermo Fisher Scientific, Waltham, MA, USA). Small hairpin RNA (shRNA) targeting TDRG1 was designed and inserted into the pGPU6/ GFP/Neo vector (Thermo Fisher Scientific). The plasmids pEGFP-C1-TDRG1 and shRNA-TDRG1 were transfected into TCam- 2 cells after reaching $70 \%$ confluence using the Lipofectamine 2000 of Thermo Fisher Scientific, according to the company's instructions.

\section{Reverse transcription polymerase chain reaction assay}

Total RNA was extracted from tissue samples and testicular seminoma TCam-2 cells using the TRIzol reagent of Takara (Dalian, People's Republic of China) following the manufacturer's protocol. Total RNA was reverse transcribed into cDNA using the PrimeScript RT Reagent Kit (Takara). Reverse transcription polymerase chain reaction (RT-PCR) was performed using the iQ5 Multicolor Real-Time PCR Detection System (Bio-Rad Laboratories Inc., Hercules, CA, USA) with SYBR Premix Ex Taq II (Takara). The PCR primer sequences for TDRGI and $\beta$-actin were as follows: TDRG1 forward primer 5'-GAAGAGGAGGGAGGCAGTCT-3' and TDRG1 reverse primer $5^{\prime}$-GGGAACCTAGACCTGGGAAG- $3^{\prime}$ and $\beta$-actin forward primer $5^{\prime}$-CATTAAGGAGAAGCTGTGCT- $3^{\prime}$ and $\beta$-actin reverse primer 5'-GTTGAAGGTAGTTTCGTGGA-3'. A melting curve analysis of the amplified products was performed at the end of each PCR cycle. The comparative C(T) method was used to quantify the expression of TDRG1 using $\beta$-actin as the normalization control.

\section{Western blot analysis}

Tissues and cell lysates were prepared using lysis buffer (50 mM Tris-HCl, pH 7.4, 150 mM NaCl, 10 mM NaF, 2 mM EDTA, $1 \%$ NP-40, and $0.1 \%$ sodium dodecyl sulfate [SDS]). Equal amounts of protein $(20 \mu \mathrm{g})$ were boiled for 5 minutes, separated by SDS-polyacrylamide gel electrophoresis on $10 \%$ polyacrylamide Tris-glycine gels, and electroblotted onto a nitrocellulose membrane. Nonspecific sites were blocked with $5 \%$ nonfat dry milk in $0.2 \%$ Tween- 20 in Trisbuffered saline (TBS-T) for 1 hour at room temperature and then probed with antibodies. After extensive washing (three times for 10 minutes each in TBS-T), horseradish peroxidaseconjugated secondary antibodies were added for 1 hour at $22^{\circ} \mathrm{C}$. Blots were again washed three times for 10 minutes each in TBS-T, and immunoreactive bands were developed by enhanced chemiluminescence. GAPDH was used as a loading control.

\section{Immunohistochemistry}

Three tissue microarray chips were purchased from Alenabio Biotechnology (Xi' an, People's Republic of China). The tissue microarray chips TE802 and TE2081 contained 80 points (from 80 testicular seminoma samples) and 138 points (from 46 testicular seminoma samples, three points per sample), respectively. TEN601 had 60 points (from 30 normal testicular tissue samples, two points per sample). Tissue microarray chips were deparaffinized with xylene and rehydrated with ethanol. Antigen retrieval was performed by placing the chips in $0.01 \mathrm{M}$ citrate buffer ( $\mathrm{pH}$ 6.0) before microwave heating for 20 minutes. Normal goat serum (10\%) was utilized for 30 minutes to block nonspecific binding sites. Subsequently, the cells were incubated with primary antibodies, including TDRG1 (1:4,000), PI3K/p85 (1:8,000), p-PI3K/p85 (1:8,000), PI3K/p110 (1:4,000), p-PI3K/p110 (1:4,000), p-Akt (1:4,000), mTOR (1:4,000), p-mTOR (Thr2446, 1:4,000), p-mTOR (Ser2448, 1:4,000), p-mTOR (Ser2481, $1: 4,000)$, and GAPDH $(1: 8,000)$, at $4^{\circ} \mathrm{C}$ overnight. The primary antibodies were visualized by adding a secondary biotin-conjugated antibody followed by an avidin/biotin/ peroxidase complex (Vectastain ABC Elite kit; Vector Laboratories lnc, Burlingame, CA, USA) and substrate (Vector NovaRED, Vectastain).

\section{Cell proliferation assay}

Cell proliferation was measured at different time points (0 hour, 12 hours, 24 hours, 48 hours, 72 hours, and 96 hours) using the 3-(4,5-dimethylthiazol-2yl)-2,5-diphenyltetrazolium bromide (MTT) assay. Cells were digested and plated in 96-well plates at a density of $5 \times 10^{3}$ cells/ well. At different time points, the medium was removed and replaced with $20 \mu \mathrm{L}$ of MTT $(5 \mathrm{mg} / \mathrm{mL})$ and incubated for 4 hours at $37^{\circ} \mathrm{C}$. Then, the supernatant was carefully removed, and dimethyl sulfoxide was added to each well to dissolve the crystals by gentle agitation for 10 minutes. For each well, the absorbance at $570 \mathrm{~nm}$ (A570) was estimated on a microplate reader (ELx800; BioTek Instruments, Inc., Winooski, VT, USA). The cell proliferation rate in each well was calculated according to the following formula: proliferation rate $(\%)=(\mathrm{A} 570$ of treated wells/A570 of untreated wells) $\times 100$.

\section{Proliferation index measurement}

After 72 hours of incubation, the cells were fixed in $70 \%$ alcohol for 30 minutes on ice. The cells were treated with 
RNase A (Sigma-Aldrich Co., St Louis, MO, USA) at $37^{\circ} \mathrm{C}$ and stained with propidium iodide in the dark for 30 minutes. DNA content was assayed by flow cytometry using a FACSCalibur instrument (BD, Franklin Lakes, NJ, USA), and a cell cycle analysis was conducted using the CellQuest software. The cell proliferation index was obtained by calculating the percentage of cells in the S, G2, and M phases.

\section{Apoptosis assay}

Apoptosis was assessed with an Annexin V-FITC/PI Apoptosis Detection Kit (Beyotime Institute of Biotechnology, Shanghai, People's Republic of China) according to the manufacturer's instructions. Briefly, after 72 hours of cultivation, cells were trypsinized, washed with phosphate-buffered saline, and stained with Annexin V and propidium iodide in the dark. The percentage of apoptotic cells was quantified using a FACSCalibur instrument.

\section{Cell adhesion assay}

Cells $\left(3 \times 10^{5}\right.$ cells $\left./ \mathrm{mL}\right)$ were seeded in 96 -well plates coated with $1 \%$ fibronectin for 2 hours and blocked with $1 \%$ bovine serum albumin for 2 hours. After 2 hours of incubation, the wells were washed with phosphate-buffered saline to remove cells that failed to adhere to the bottom, and cells were fixed in paraformaldehyde for 30 minutes. After 2 hours of staining with $100 \mu \mathrm{L}$ of $0.5 \%$ crystal violet, $100 \mu \mathrm{L}$ of $2 \%$ SDS was added to each well to determine the number of adherent cells based on absorbance at $570 \mathrm{~nm}$.

\section{Cell invasion assay}

A cell invasion assay was performed using Transwell chambers (Corning Incorporated, Corning, NY, USA) with $8 \mu \mathrm{m}$ pore inserts coated with Matrigel (Sigma-Aldrich Co.). Cell suspensions were seeded in the upper chamber, and $500 \mu \mathrm{L}$ of Dulbecco's Modified Eagle's Medium containing fetal bovine serum was added to the lower chamber. The noninvading cells were removed with a cotton-tipped swab after 24 hours of incubation, and the invading cells on the bottom surface of the membrane were stained with $0.1 \%$ crystal violet. The invading cells were quantified by counting ten random fields at $\times 200$ magnification.

\section{Statistical analysis}

The data are expressed as the mean \pm standard deviation. A statistical analysis of the data was performed using SPSS 17.0 (SPSS Inc., Chicago, IL, USA). Statistical significance was determined using an analysis of variance followed by
Student's $t$-tests. A value of $P<0.05$ was considered to indicate a statistically significant difference.

\section{Results \\ TDRG I was upregulated in testicular seminoma tissues}

We examined whether testicular seminoma tissues differ from normal testicular tissues with respect to TDRG1 expression in both gene and protein levels via RT-PCR and Western blot assays, respectively. The levels of gene and protein TDRG1 expression were, respectively, 68\% $(P=0.037)$ and $90 \%(P=0.029)$ higher in testicular seminoma tissues $(n=33)$ than in normal testicular tissues $(n=33)$ (Figure 1A and B). In the immunohistochemistry analysis comparing the average values of two testicular seminoma chips $(n=218)$ with those of a normal testicular tissue chip $(\mathrm{n}=60), T D R G 1$ expression was 2.4 times higher in testicular seminoma tissues than in the normal testicular tissue $(P=0.014$, Figure $1 C)$. These data provide clear evidence that TDRG1 is upregulated in testicular seminoma tissues.

\section{TDRG I modulated the proliferation and invasion of testicular seminoma TCam- 2 cells}

To gain insight into the functions of TDRG1 in testicular seminomas, the gene was artificially upregulated or downregulated in testicular seminoma TCam-2 cells. Transfection with shRNA-TDRG1 strongly inhibited TDRG1 expression at both the gene and the protein levels $(P<0.01)$, as shown in Figure 2A and B, respectively. Conversely, transfection with the pEGFP-C1-TDRG1 vector (TDRG1 expression vector) resulted in the overexpression of $T D R G 1$, and the gene and protein expression levels were approximately four and two times higher than those of control cells $(P<0.01$ and $P=0.022$ ). The expression of TDRG1 was essentially unaffected by transfection with the $\mathrm{pEGFP-C1}$ vector. Also, administration of LY294002 or IGF-1 exhibited no effect on the TDRG1 protein expression level in the testicular seminoma TCam-2 cells.

Cell proliferation was initially detected using MTT tests at different time points (Figure 3A). Inhibiting the expression of TDRG1 decreased the rate of proliferation for testicular seminoma TCam- 2 cells. We analyzed the differences in the cell proliferation rate between each group at 72 hours and 96 hours and found that TDRG1 downregulation inhibited the cell proliferation rate markedly at both time points $(P=0.033$ and $P=0.029$ ). The overexpression of $T D R G 1$ via transfection 
A

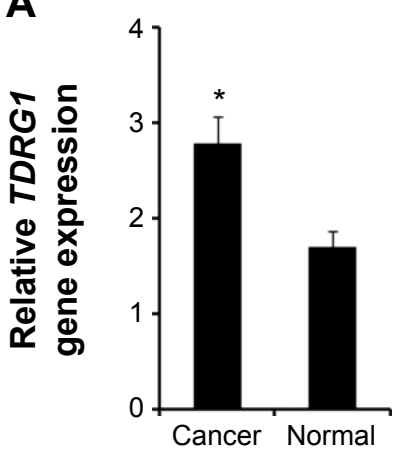

Bi
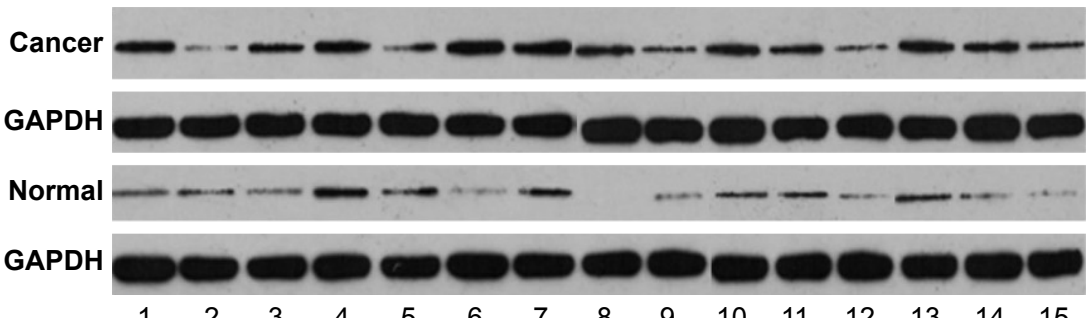

Bii

$\mathbf{C i}_{\mathrm{i}}$

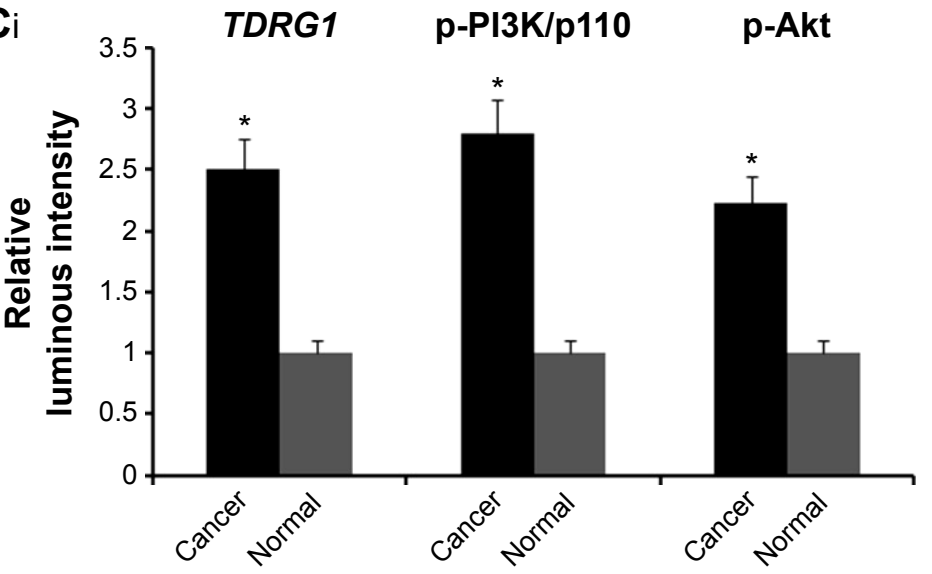

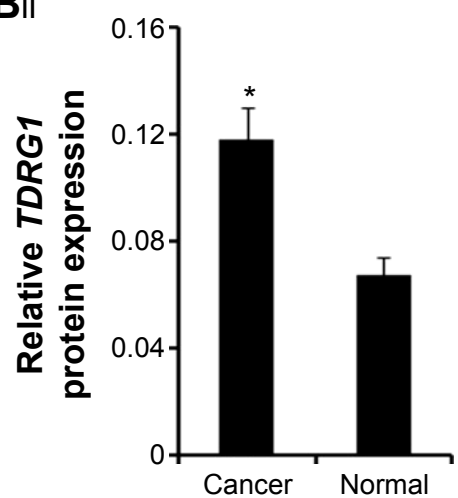

$\mathrm{C}_{\mathrm{ii}}$

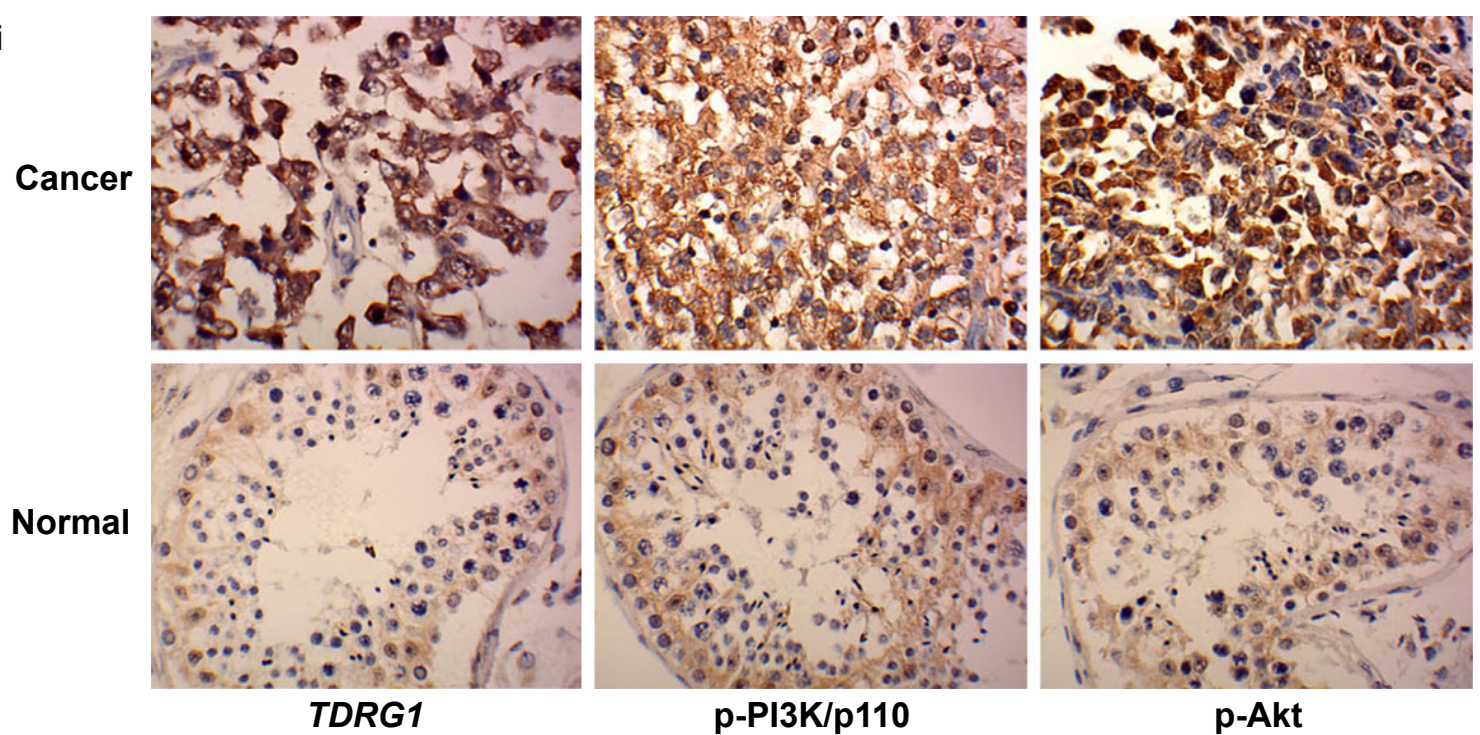

Figure I TDRGI is significantly upregulated in testicular seminoma tissues than in normal testicular tissues.

Notes: (A) RT-PCR showed that the relative gene expression level of TDRGI is significantly higher in testicular seminoma tissues than in normal testicular tissues. (Bi) Representative Western blotting images that are associated with TDRGI and GAPDH protein expression in both testicular seminoma and normal testicular tissues. (Bii) Western blotting showed that the relative protein expression level of TDRGI is significantly higher in testicular seminoma tissues than in normal testicular tissues. RT-PCR and Western blotting were performed to detect the expression levels of TDRGI in both testicular seminoma tissues $(\mathrm{n}=33)$ and normal testicular tissues $(\mathrm{n}=33)$. ( $\mathrm{Ci})$ Immunohistochemical staining pictures of both testicular seminoma and normal testicular tissue samples with anti-TDRGI, anti-p-PI3K/pI I0, and anti-p-Akt antibodies. (Cii) The protein expression levels of TDRGI, p-PI3K/pI I0, and p-Akt are significantly upregulated in testicular seminoma tissues compared to normal testicular tissues. Protein expression levels of TDRGI, $\mathrm{p}-\mathrm{PI} 3 \mathrm{~K} / \mathrm{pl}$ I0, and p-Akt were detected by an immunohistochemistry assay. The tissue microarray chips TE802 and TE208I contained 80 points (from 80 testicular seminoma tissue samples) and 138 points (from 46 testicular seminoma tissue samples, three points per sample), respectively. TEN60I had 60 points (from 30 normal testicular tissue samples, two points per sample). Bars representing cancer are averages of data from 218 points $(80+138)$ in two tissue microarray chips. Bars representing the normal tissue samples are the average of data from 60 points in a tissue microarray chip. ${ }^{*} P<0.05$ vs control group. Cancer: testicular seminoma; normal: normal testicular tissues.

Abbreviations: TDRGI, testis development-related gene I; RT-PCR, reverse transcription polymerase chain reaction; PI3K, phosphoinositide-3 kinase; GADPH, glyceraldehyde-3-phosphate dehydrogenase. 
A

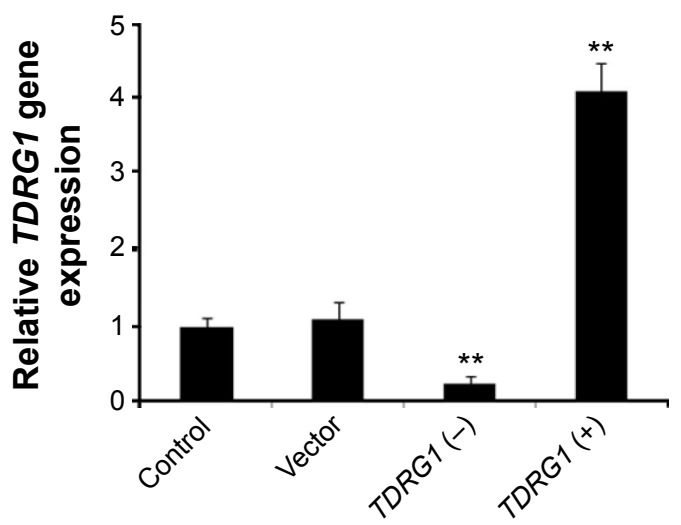

B
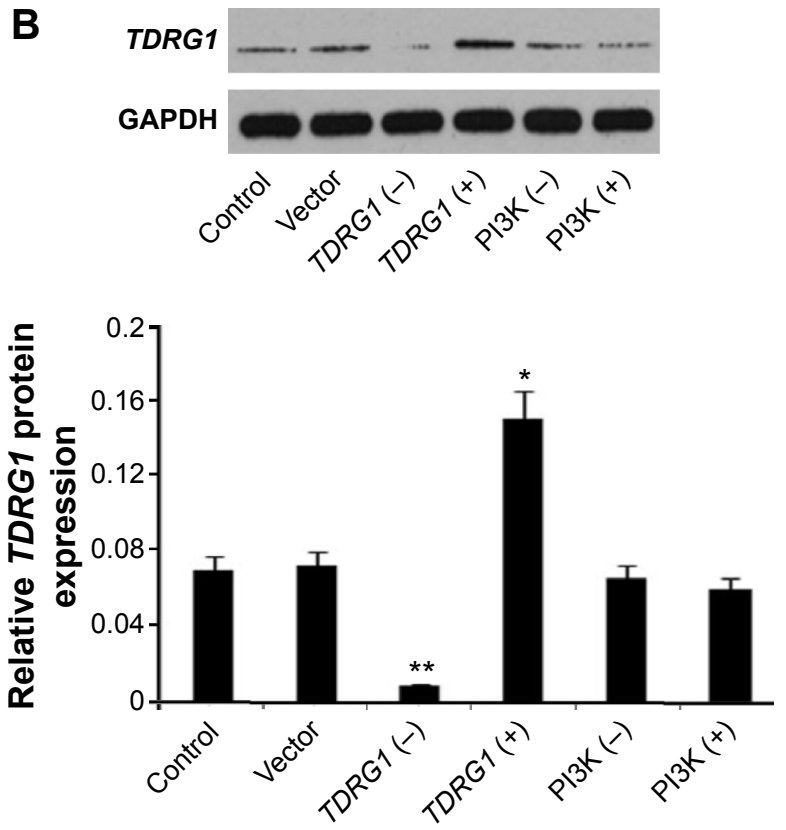

Figure 2 Relative TDRGI expression in both gene and protein levels of seminoma TCam- 2 cells after different treatments.

Notes: (A) Relative gene expression level of TDRGI in seminoma TCam-2 cells was remarkably upregulated and downregulated after the gene was knocked out and overexpressed, respectively, on the basis of RT-PCR $(n=5)$. (B) Relative protein expression level of TDRGI in seminoma TCam-2 cells was remarkably upregulated and downregulated after the gene was knocked out and overexpressed, respectively, but was unaffected after treatment with LY294002 or insulin-like growth factor-I on the basis of Western blotting. $* P<0.05$ and $* * P<0.01$ vs control group. Vector: enhanced green fluorescent protein plasmid-CI vector.

Abbreviations: TDRGI, testis development-related gene I; RT-PCR, reverse transcription polymerase chain reaction; PI3K, phosphoinositide-3 kinase; TDRGI (-), the knockout of TDRGI; TDRGI (+), the overexpression of TDRGI; PI3K (-), the inhibition of PI3K by LY294002; PI3K (+), the activation of PI3K by insulin-like growth factor-I.

with the TDRG1 expression vector accelerated cell proliferation relative to control cells, but this difference was not statistically significant; the biggest difference in the cell proliferation rate between treated and control cells was observed at 72 hours.

The reduction in the cell proliferation rate could be explained by a slower progression of cells through the cell cycle and/or increased cell death. ${ }^{22}$ To evaluate these mechanisms, we investigated the cell cycle and the apoptosis rate at
72 hours. The cell cycle is subdivided into the G1, S, G2, and M phases, which are controlled and coordinated by several "checkpoints." A major checkpoint, also known as a restriction point, has been identified before the G1-S transition, after which a cell is committed to division. ${ }^{23,24}$ Thus, the cell proliferation index was evaluated by estimating the percentage of cells in S, G2, and M phases. As shown in Figure 3B, TDRG1 knockout significantly decreased the TCam-2 cell proliferation index $(P=0.039)$, indicating that far more cells were arrested in the G1 phase. We also stained TCam-2 cells with Annexin V/PI, which specifically labels apoptotic cells, to estimate the apoptosis rate. ${ }^{24}$ The inhibition of TDRG1 expression predominantly augmented the apoptosis rate $(P<0.01$, Figure 3C), suggesting that the modulation of cell proliferation by TDRG1 is related to the regulation of cell fate (survival or apoptosis). It should be noted that neither the proliferation index nor the apoptosis rate was significantly affected by the overexpression of TDRG1.

The impact of TDRG1 on cancer cell invasion was tested by cell adhesion and cell invasion assays at 72 hours. Cancer cells, especially the highly metastatic types, are believed to have enhanced adhesion ability that often facilitates the migration of the cells to a new site to establish new tumors in the body. In cell adhesion assay, cancer cells with strong adhesion ability can tightly bind to the plates and avoid to be washed away by PBS, thus estimating the numbers of cells left on the plates leads to the understanding of adhesion ability of cancer cells. Cell invasion assays monitored the cells that passed through the transwell, thereby reflecting the ability of the cells to move through the extracellular matrix into the neighboring tissues in body. Both adherence and invasion are crucial cancer cell properties that reflect the invasive ability of testicular seminoma TCam- 2 cells. Our data indicated that the downregulation of TDRG1 inhibited the adhesion and invasion capabilities of TCam- 2 cells ( $P=0.027$, Figure 4A, and $P=0.031$, Figure 4B, respectively). By contrast, the overexpression of TDRG1 showed the opposite results, dramatically enhancing cell adhesion and invasion abilities $(P=0.025$ and $P=0.029$, respectively). These data indicated that the invasion of testicular seminoma TCam- 2 cells is positively correlated with the expression level of TDRG1.

\section{$\mathrm{PI} 3 \mathrm{~K} / \mathrm{Akt} / \mathrm{mTOR}$ signaling cascades regulated the proliferation and invasion abilities of testicular seminoma}

\section{TCam- 2 cells}

$\mathrm{PI} 3 \mathrm{~K} / \mathrm{Akt} / \mathrm{mTOR}$ signaling cascade is classically involved in cell proliferation and invasion in many cancer types. ${ }^{25-27}$ 


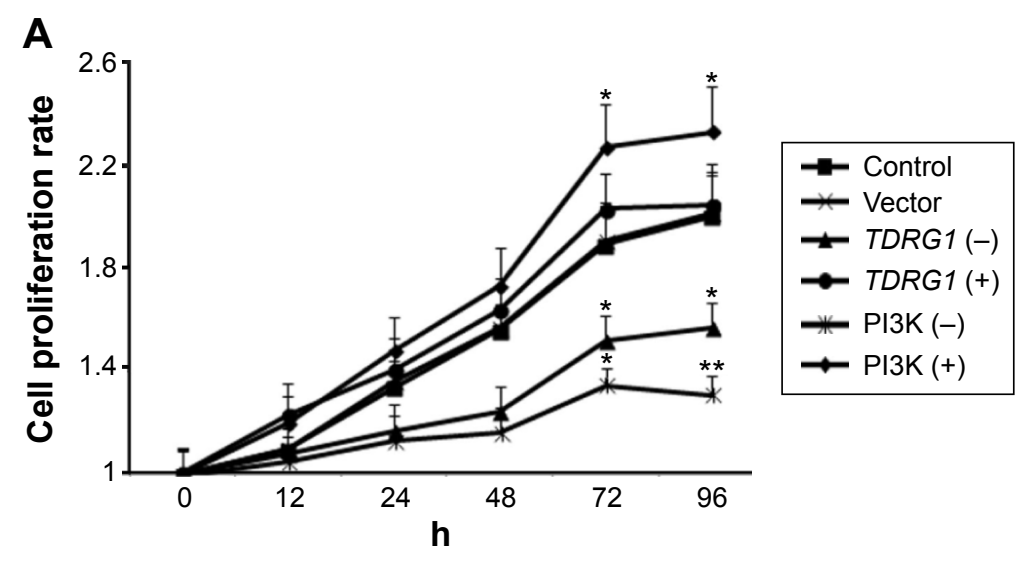

B i
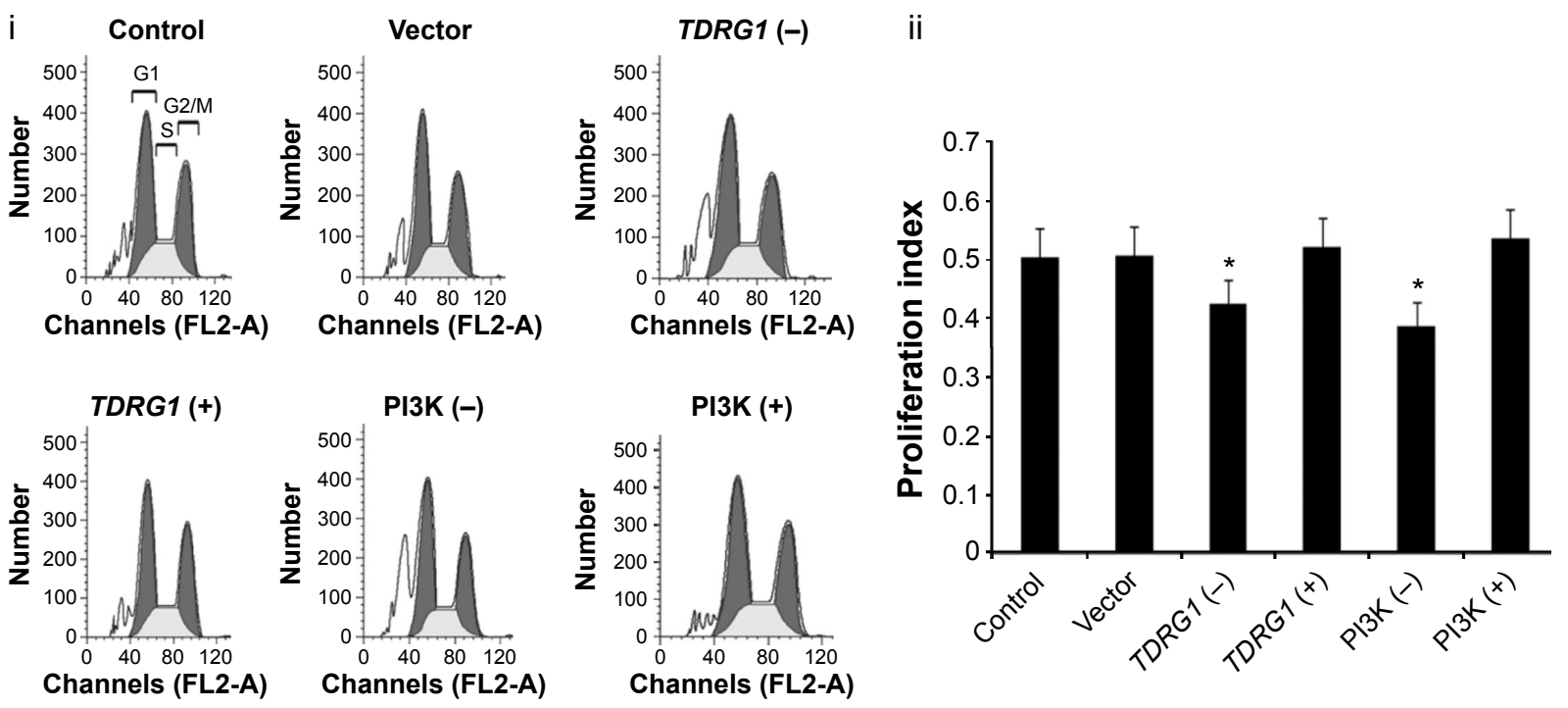

C
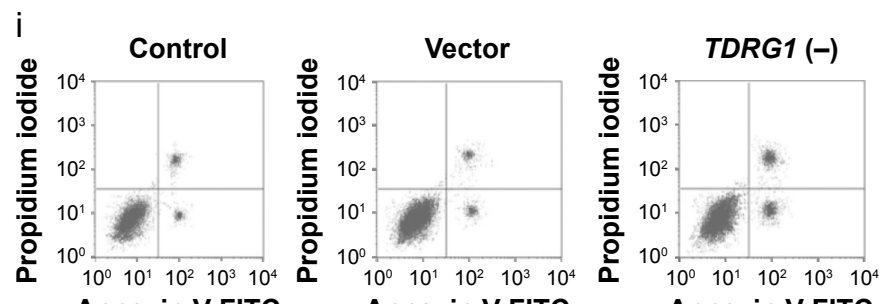

ii

Annexin V-FITC

Annexin V-FITC
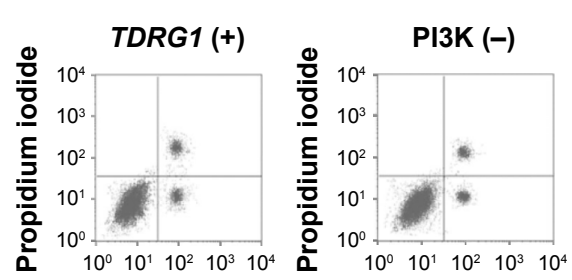

Annexin V-FITC

Annexin V-FITC
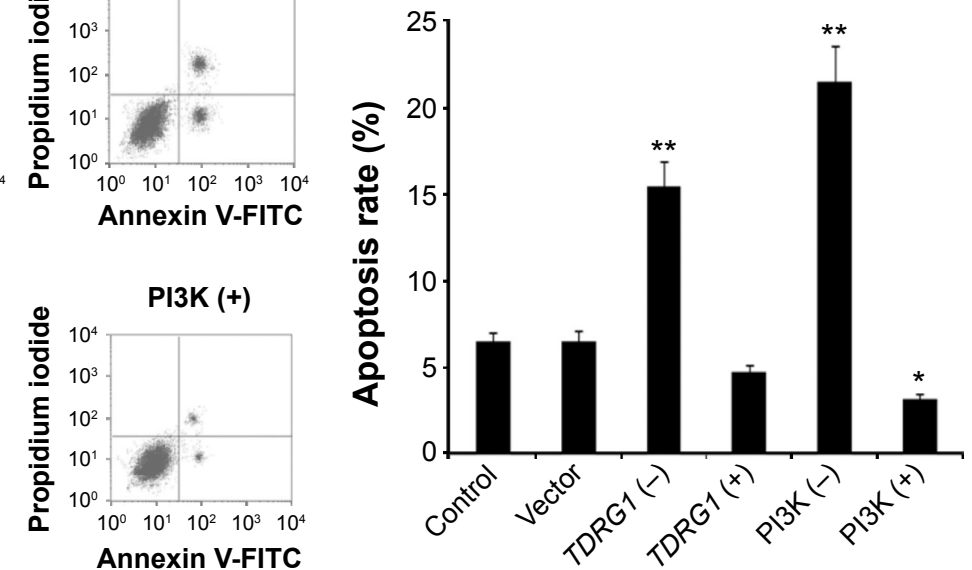

Figure 3 The effect of TDRGI - and PI3K-mediated signaling on the cell proliferation rate, cell proliferation index, and apoptosis rate.

Notes: (A) Cells with both TDRGI knockout and PI3K inhibition exhibited decreased cell proliferation. (Bi and Bii) Cells with both TDRGI knockout and PI3K inhibition exhibited decreased cell proliferation index. (Ci and $\mathbf{C i i}$ ) Cells with both TDRGI knockout and PI3K inhibition exhibited increased apoptosis rate. Seminoma TCam-2 cells were subjected to different treatments: the TDRGI knockout, TDRGI overexpression, PI3K inhibition (LY294002 administration), or PI3K activation (IGF-I administration). The cell proliferation rate was assessed using MTT kits at different time points after the treatments, and cell proliferation index and apoptosis rate were evaluated using flow cytometry at $72 \mathrm{~h}$. The cell proliferation index was obtained by calculating the percentage of cells in the $\mathrm{S}, \mathrm{G} 2$, and $\mathrm{M}$ phases. $* \mathrm{P}<0.05$ and $* * P<0.0 \mathrm{I}$ vs control group. Vector: enhanced green fluorescent protein plasmid-Cl vector.

Abbreviations: TDRGI, testis development-related gene I; PI3K, phosphoinositide-3 kinase; IGF-I, insulin-like growth factor-I; MTT, 3-(4,5-dimethylthiazol-2yI)-2,5diphenyltetrazolium bromide; h, hours; TDRGI (-), the knockout of TDRGI; TDRGI (+), the overexpression of TDRGI; PI3K (-), the inhibition of PI3K by LY294002; PI3K (+), the activation of PI3K by IGF-I; FL2-A, fluorescence 2 area; FITC, fluorescein isothiocyanate. 


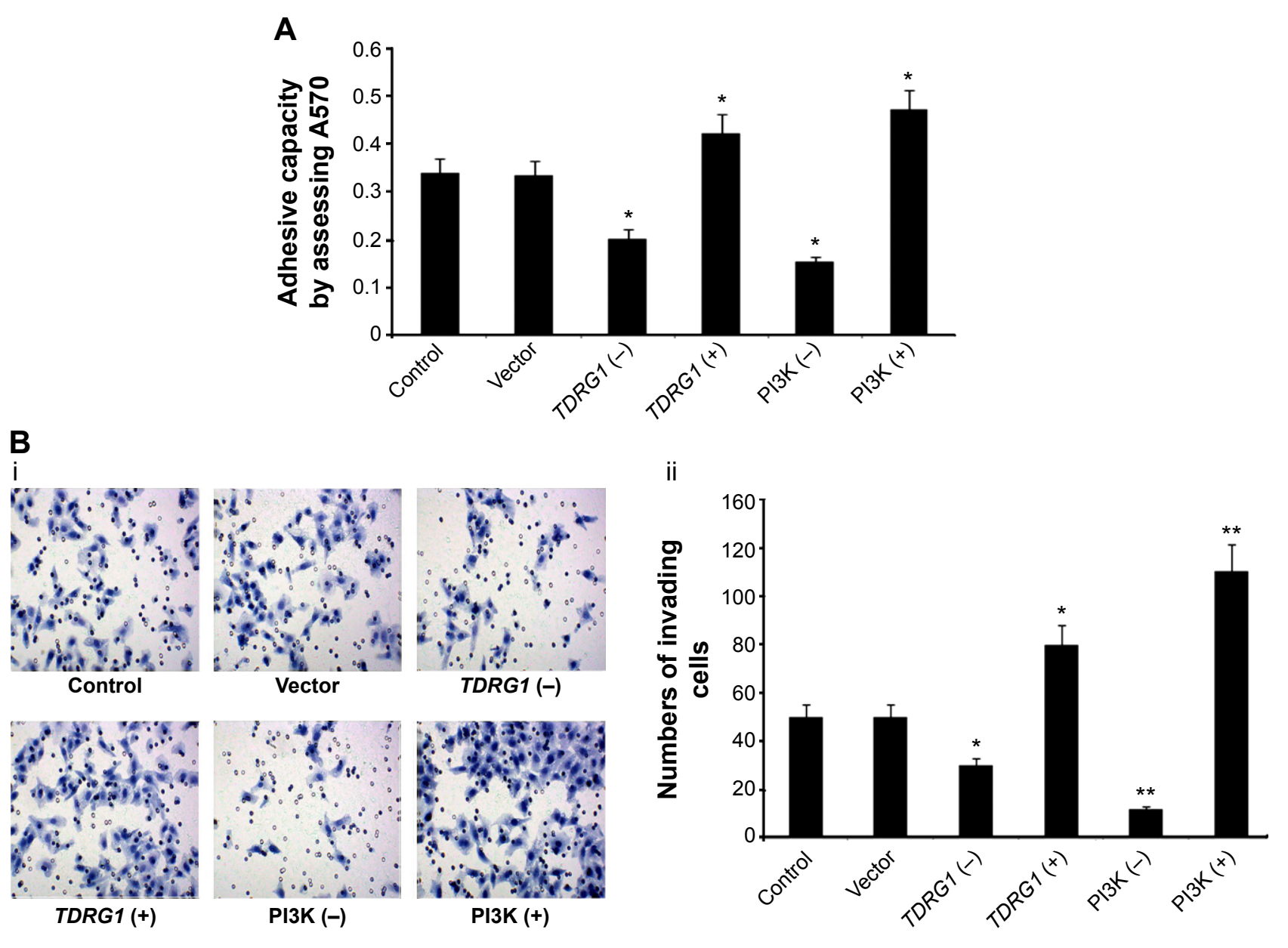

Figure 4 The effect of TDRGI - and PI3K-mediated signaling on the cell adhesive capacity and cell invasion.

Notes: (A) The cell adhesive capacity was increased in the cells with both TDRGI knockout and PI3K inhibition and decreased in the cells with both TDRGI overexpression and PI3K activation. (Bi) Pictures of cell passing through transwells. (Bii) The cell invasion was increased in the cells with both TDRGI knockout and PI3K inhibition and decreased in the cells with both TDRGI overexpression and PI3K activation. Seminoma TCam-2 cells were subjected to different treatments: the TDRGI knockout, TDRGI overexpression, PI3K inhibition (LY294002 administration), or PI3K activation (IGF-I administration). $* P<0.05$ and $* * P<0.01$ vs control group. Vector: enhanced green fluorescent protein plasmid-CI vector.

Abbreviations: TDRGI, testis development-related gene I; PI3K, phosphoinositide-3 kinase; IGF-I, insulin-like growth factor-I; TDRGI (-), the knockout of TDRGI; TDRGI (+), the overexpression of TDRGI; PI3K (-), the inhibition of PI3K by LY294002; PI3K (+), the activation of PI3K by IGF-I.

To determine the effects of the PI3K/Akt/mTOR signaling pathway on testicular seminoma, we pharmacologically inhibited or activated the pathway via treatments with LY294002 (a specific inhibitor) or IGF-1 (an activator). Western blotting showed the efficacy of these two kinds of substances (Figure 5). The treatment with LY294002 inhibited the expression of PI3K/p110 $(P=0.025), \mathrm{p}-\mathrm{PI} 3 \mathrm{~K} / \mathrm{p} 110$ $(P<0.01)$, p-Akt $(P<0.01)$, and p-mTOR (S2448) $(P<0.01)$, whereas pharmacological stimulation with IGF-1 caused a marked elevation in the phosphorylation levels of PI3K/p110 $(P=0.031)$, Akt $(P=0.035)$, and mTOR (S2448) $(P<0.01)$.

The pharmacological regulation of the PI3K/Akt/mTOR signaling pathway resulted in changes in cell proliferation and invasion. Based on an MTT cell proliferation test (Figure 3A), treatment with LY294002 decreased cell proliferation; specifically, the cell proliferation rate was remarkably lower than that of the control at 72 hours $(P=0.029)$ and 96 hours $(P<0.01)$. The IGF-1 treatment group showed the highest cell proliferation curve among all treatment groups. The cell proliferation rates at 72 hours and 96 hours were significantly elevated in this group ( $P=0.025$ and $P=0.031$, respectively). To compare the effects of the PI3K/Akt/mTOR signaling pathway with those of TDRG1, the cell proliferation index, apoptosis rate, and cell invasion capability in response to changes in PI3K/Akt/mTOR signaling were examined at 72 hours. Similar to the effects of TDRG1 downregulation, LY294002 treatment resulted in a dramatic reduction in the cell proliferation index of TCam-2 cells ( $P=0.033$, Figure 3B). It also induced a highly significant increase in the apoptosis rate $(P<0.01$, Figure $3 C)$. No notable effect on the cell proliferation index was detected for IGF-1 treatment, but it significantly elevated the apoptosis rate $(P=0.039$, Figure $3 C)$. 


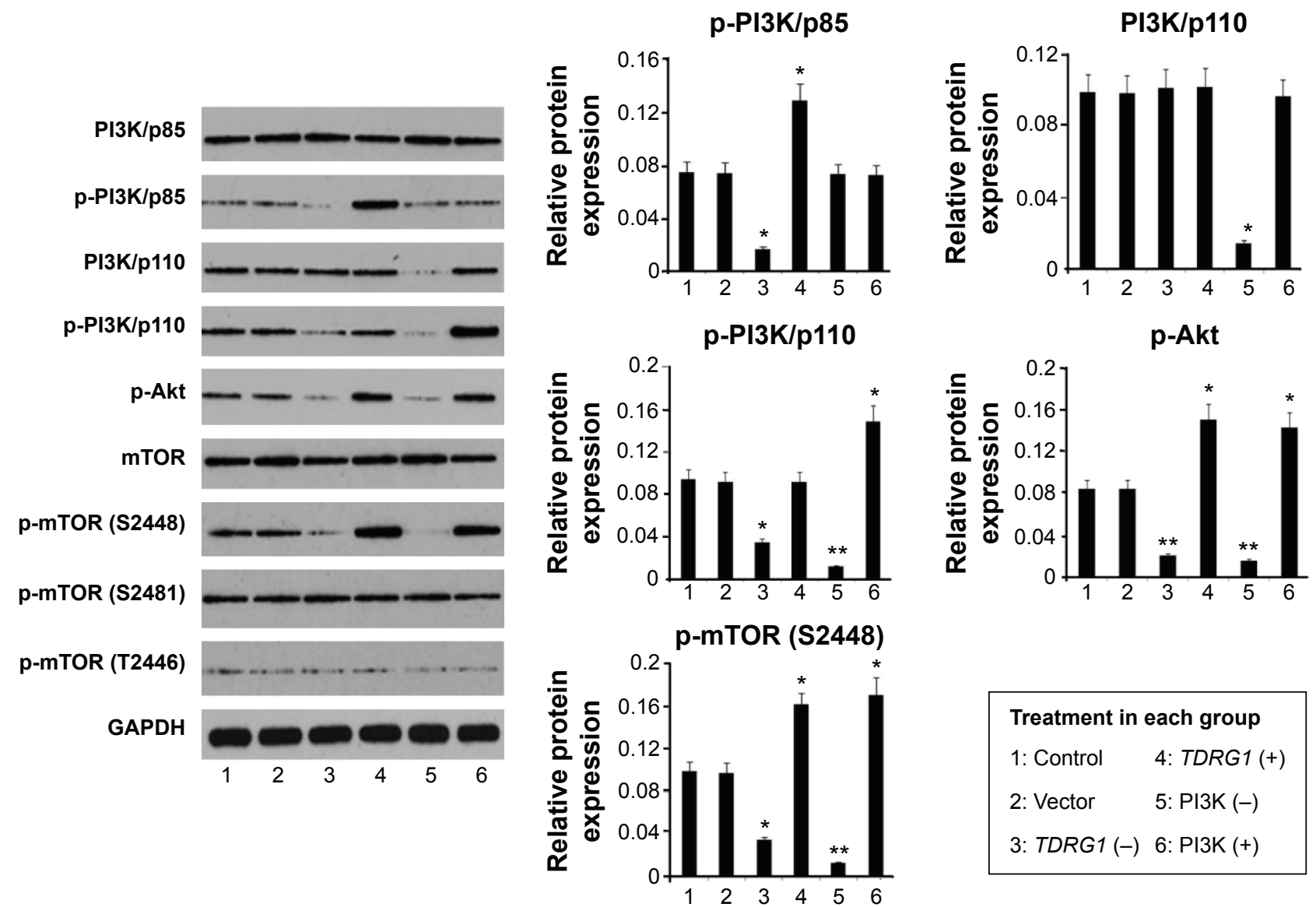

Figure 5 Expression levels of various proteins in seminoma TCam-2 cells after different treatments based on a Western blot assay.

Notes: Seminoma TCam-2 cells were subjected to different treatments: the TDRGI knockout, TDRGI overexpression, PI3K inhibition (LY294002 administration), or PI3K activation (IGF-I administration). Inhibiting TDRGI causes a dramatic reduction in the protein expression levels of $\mathrm{p}-\mathrm{PI} 3 \mathrm{~K} / \mathrm{p} 85$, $\mathrm{p}-\mathrm{PI3K} / \mathrm{p}$ II0, $\mathrm{p}-\mathrm{Akt}$, and $\mathrm{p}-\mathrm{mTOR}$ (S2448) but not PI3K/p85, PI3K/pI I0, mTOR, p-mTOR (S248I), and p-mTOR (T2446). In contrast, overexpression of TDRGI promoted the expression of p-PI3K/p85, p-Akt, and p-mTOR (S2448). The treatment with LY294002 inhibited the expression of PI3K/pI I0, p-PI3K/p I I0, p-Akt, and p-mTOR (S2448), while pharmacological stimulation with IGF-I caused a marked elevation in the phosphorylation levels of PI3K/pII0, Akt, and mTOR (S2448). $* P<0.05$ and $* * P<0.01$ vs control group. Vector: enhanced green fluorescent protein plasmid-Cl vector.

Abbreviations: TDRGI, testis development-related gene I; PI3K, phosphoinositide-3 kinase; IGF-I, insulin-like growth factor-I; mTOR, mammalian target of rapamycin; TDRGI (-), the knockout of TDRGI; TDRGI (+), the overexpression of TDRGI; PI3K (-), the inhibition of PI3K by LY294002; PI3K (+), the activation of PI3K by IGF-I; GADPH, glyceraldehyde-3-phosphate dehydrogenase.

The cell adhesion capacity responded similarly to changes in the signaling pathway and changes in the expression level of TDRG1. Specifically, cell adhesion was positively correlated with PI3K/Akt/mTOR signaling (Figure 4A). After signaling was repressed by LY294002, the cell adhesion capacity of TCam-2 cells was remarkably attenuated $(P=0.021)$. In contrast, it was highly strengthened by IGF-1 simulation of PI3K/Akt/mTOR signaling. A cell invasion assay showed a similar pattern. As shown in Figure 4B, the number of TCam-2 cells that passed through the Transwell membrane decreased to $\sim 30 \%$ of the number of control cells that passed through the membrane after LY294002-mediated inhibition of PI3K/Akt/mTOR signaling $(P<0.01)$. Activation of PI3K/Akt/mTOR signaling had the opposite effect, suggesting that $\mathrm{PI} 3 \mathrm{~K} / \mathrm{Akt} / \mathrm{mTOR}$ signaling modulates cell invasion.

\section{TDRGI regulated $\mathrm{PI} 3 \mathrm{~K} / \mathrm{Akt} / \mathrm{mTOR}$ signaling cascades}

Aberrant activation of the PI3K/Akt/mTOR signaling cascade is commonly observed in diverse cancer types ${ }^{25-27}$ Consistent with this, in our immunohistochemistry test (Figure 1C), $T D R G 1$ upregulation was associated with approximately twofold increases in the protein expression levels of both p-PI3K/ p110 and p-Akt in testicular seminoma relative to normal testicular tissues $(P=0.013$ and $P=0.019)$, implying a link between TDRG1 and the PI3K/Akt signaling pathway. The Western blot results showed that RNA interference inhibiting TDRG1 causes a dramatic reduction in the protein expression levels of p-PI3K/p85, p-PI3K/p110, p-Akt, and p-mTOR (S2448) but not PI3K/p85, PI3K/p110, mTOR, p-mTOR (S2481), and p-mTOR (T2446). In contrast, overexpression of TDRG1 promoted the expression of $\mathrm{p}-\mathrm{PI} 3 \mathrm{~K} / \mathrm{p} 85$, p-Akt, 
and p-mTOR (S2448). These data indicated that the PI3K/ Akt/mTOR signaling pathway is regulated by TDRG1.

\section{Discussion}

Testicular seminoma arises from the dedifferentiation and/or malignant transformation of spermatogenic cells..$^{28,29}$ Coincidently, the human testis-specific gene TDRG1 is primarily expressed in spermatogenic cells of the seminiferous tubule but not in the testicular Sertoli cells. ${ }^{9}$ Its expression level is the highest during puberty and decreases with age; it is not expressed during the fetal period. ${ }^{9}$ The time at which $T D R G 1$ expression is the highest coincides with the period during which testicular seminoma occurrence is maximal (between 15 years and 35 years of age). Thus, we speculated that TDRG1 contributes to the occurrence and/or development of testicular seminoma. Moreover, based on our PCR, Western blotting, and immunohistochemistry results, TDRG1 expression at both the gene and the protein levels was significantly higher in testicular seminoma tissues than in normal testicular tissues. This finding supported our hypothesis.

Functional research on TDRG1 with respect to seminoma regulation was performed in seminoma TCam- 2 cells. The knockout of TDRG1 strikingly diminished cell proliferation by arresting the cell cycle and reducing apoptosis, and reduced cell invasion by repressing the cell adhesion and invasion capacities. TDRG1 serves as a key modulator that not only promotes seminoma cell proliferation and growth but also confers an aggressive seminoma phenotype by facilitating adhesion and invasion to other tissues. However, artificially elevating $T D R G 1$ expression via transfection with a $T D R G 1$ expression vector only resulted in marginal differences in cell proliferation, although it remarkably increased cancer cell adhesion and invasion. A possible explanation for this observation is that underlying TDRG1 upregulation in the seminoma already exerted positive effects on cell proliferation. Additional upregulation of TDRG1 may exceed its ability to increase cell proliferation. PI3K-mediated signaling pathways, in addition to the regulation of normal cell processes, are associated with cancer formation and progression in diverse cancer types, such as pulmonary, pancreatic, colorectal, gastric, and ovarian. ${ }^{25-27,30,31}$ To examine whether the same mechanisms related to PI3K signaling pathways are involved in seminomas, PI3K-mediated signaling was pharmacologically inhibited using LY294002. Various characteristics contributing to seminoma cell proliferation and invasion that were repressed by LY294002 were similar to those inhibited by TDRG1 knockout. Treatment with the specific PI3K activator IGF-1, which interacts with IGF-1 receptors to initiate PI3K-stimulating signals via the adaptor molecule insulin receptor substrate- $1,{ }^{32}$ promoted the proliferation and invasion of seminoma cells, confirming that the PI3K pathway plays a positive and critical role in seminoma development.

PI3K is a heterodimer composed of the regulatory subunit $\mathrm{p} 85$ and catalytic subunit $\mathrm{p} 110$. The activation of PI3K requires the recruitment of PI3K/p85 from the cytoplasm and localization on the cell membrane to bind to a phosphotyrosine residue of an adapter protein, such as insulin receptor substrate- $1 .{ }^{33}$ During this process, the phosphorylation of PI3K/p85 regulates the activation of PI3K, although the proximal mechanisms are not well understood. The Ser-83 residue on $\mathrm{p} 85$ is phosphorylated by protein kinase $\mathrm{A}$, triggering increased PI3K activity. ${ }^{34}$ Additionally, the 14-3-3 proteins that regulate many pathways involved in cell transformation in eukaryotic organisms bind to $\mathrm{p} 85$ at the phospho-serine 83 motif in the N-terminal domain of $\mathrm{p} 85$, and this is largely responsible for $14-3-3 \zeta$-mediated PI3K membrane recruitment and activation. ${ }^{35}$ In our study, PI3K/ p85 phosphorylation was positively correlated with the expression of TDRG1. Knocking out TDRG1 reduced PI3K/ p85 phosphorylation. Ectopic expression of TDRG1 had the opposite effect. Combined with previous findings, our data suggested that TDRG1 positively regulates the activity of PI3K via the modulation of PI3K/p85 phosphorylation.

The catalytic subunit PI3K/p110, responsible for the synthesis of phosphatidylinositol second messengers via catalysis of PIP2 (phosphoinositol-4,5-bisphosphate) into PIP3 (phosphoinositol 3,4,5-trisphosphate), is also thought to be related to the activation of the PI3K pathway; in $>30 \%$ of solid tumors, PI3K/p100 mutation leads to unregulated PI3K activity. ${ }^{11,14,36,37}$ Additionally, both the PI3K/p110 gene and the protein are amplified substantially in ovarian and breast tumors. ${ }^{38,39}$ Interestingly, in our study, PI3K/p110 showed minor differences between testicular seminoma tissues and normal testicular tissues (data not shown), but phosphorylated PI3K/p110 was significantly elevated in seminoma tissues. Phosphorylated PI3K/p110 was substantially reduced in seminoma TCam-2 cells after TDRG1 was knocked out, suggesting that the phosphorylation of $\mathrm{PI} 3 \mathrm{~K} / \mathrm{p} 110$ is related to TDRG1. The effect of phosphorylated PI3K/p110 on $\mathrm{PI} 3 \mathrm{~K}$ activity is not clear, but our results suggest a positive correlation between the phosphorylation level of PI3K/ p110 and the activation of PI3K via LY294002 and IGF-1; accordingly, PI3K/p110 phosphorylation likely contributes to PI3K activation. 
Akt, a serine/threonine kinase, is a central mediator of PI3K cascades. Akt activation requires activated PI3K, which converts PIP2 into PIP3 to recruit Akt to the cell membrane by binding their pleckstrin homology domains; this leads to the phosphorylation of Akt, which is essential for subsequent Akt activation. ${ }^{40}$ Our immunohistochemistry assays indicated that the Akt phosphorylation level is much higher in seminoma tissues than in normal testicular tissues, and Akt overactivation is common in seminomas. Furthermore, Akt phosphorylation in TCam-2 cells showed a similar pattern to the gene and protein expression of $T D R G 1$, irrespective of whether TDRG1 was knocked out or overexpressed. This suggests that the activity of Akt in seminomas is influenced by TDRG1. The pattern of TDRG1 regulation of Akt activity is similar to that of TDRG1 regulation of PI3K, which raises the possibility that TDRG1 exerts an effect on Akt as consequence of its regulation of PI3K.

mTOR is regulated by PI3K/Akt signaling, and a particular mTOR site, $\mathrm{Ser}^{2448}$, is phosphorylated in response to PI3K/ Akt activation. ${ }^{26}$ PI3K activation via LY294002 resulted in the activation of PI3K/Akt signaling and increased the phosphorylation of mTOR at the $\mathrm{Ser}^{2448}$ site. IGF-1, in contrast, strongly inhibits the activity of the PI3K/Akt pathway and reduces the phosphorylated form of mTOR at the $\mathrm{Ser}^{2448}$ site. Aside from $\mathrm{Ser}^{2448}$, $\mathrm{Thr}^{2446}$, and $\mathrm{Ser}^{2481}$ are also phosphorylation sites that are regulated in mTOR. $\mathrm{Thr}^{2446}$ is a major adenosine monophosphate kinase-stimulated phosphorylation site. ${ }^{41}$ $\mathrm{Ser}^{2481}$ is an autophosphorylation site in mTOR that is regulated by rapamycin and amino acid withdrawal. ${ }^{42}$ In this study, phosphorylated mTOR at $\mathrm{Ser}^{2448}$ was elevated in response to TDRG1 overexpression. The downregulation of TDRG1 effectively suppressed the phosphorylation of mTOR at $\mathrm{Se}^{2448}$. However, TDRG1 failed to modulate mTOR phosphorylation at $\mathrm{Ser}^{2481}$ and $\mathrm{Thr}^{2446}$. These data further indicate that TDRG1 stimulates the PI3K/Akt signaling pathway in seminomas, and this subsequently activates mTOR-mediated signaling.

The PI3K/Akt/mTOR signaling pathway is widely known to play a crucial role in carcinogenesis and cancer progress. Mutations in some loci of the PI3K/Akt/mTOR pathway are frequently found in head and neck tumors based on the analyses of a large number of related patients, and these mutations lead to the aberrant activation of $\mathrm{PI} 3 \mathrm{~K} / \mathrm{Akt} / \mathrm{mTOR}$ and subsequent malignant transformation of head and neck tumor cells. ${ }^{43}$ In endometrial cancer, the overactivation of the PI3K/Akt/mTOR pathway plays an important role in cell growth and survival. ${ }^{44}$ Similar findings have been reported in ovarian, colorectal, and prostate cancers. ${ }^{25-27}$ In the present study, we demonstrated that the PI3K/Akt/mTOR pathway is involved in the regulation of diverse hallmarks of seminomas, including cell proliferation, apoptosis, adhesion, and invasion. More importantly, the PI3K/Akt/mTOR signaling pathway is involved in signaling downstream of TDRG1, and this may be particularly relevant to the development of seminomas. It should be noted that the physiological role of TDRG1 in testis is unclear, this problem will be addressed in our following research.

\section{Conclusion}

This is the first demonstration that TDRG1 acts as an oncogene in seminomas. This is evidenced by its dramatic upregulation in seminomas, based on PCR, Western blotting, and immunohistochemistry tests, as well as by its positive regulation of cell proliferation and invasion. Additionally, the $\mathrm{PI} 3 \mathrm{~K} / \mathrm{Akt} / \mathrm{mTOR}$ signaling pathway plays an important role in seminomas; the proliferation and invasion of seminoma cells were strongly influenced by the activation and suppression of PI3K/Akt/mTOR signaling. More importantly, the phosphorylation levels of $\mathrm{PI} 3 \mathrm{~K} / \mathrm{p} 85$, PI3K/p110, and Akt as well as the phosphorylation sites of mTOR at $\mathrm{Se}^{2448}$, $\mathrm{Ser}^{2481}$, and $\mathrm{Thr}^{2446}$ provide powerful evidences that TDRG1 is primarily related to seminoma development via the PI3K/ Akt/mTOR signaling pathway.

\section{Acknowledgment}

This study was supported by the National Nature Science Foundation of China (no 81372181).

\section{Disclosure}

The authors report no conflicts of interest in this work.

\section{References}

1. Ismaili N, Naciri S, Afqir S, et al. A rare case of advanced testicular seminoma in a 78-year-old man managed successfully with carboplatin based chemotherapy: a case report. Cases J. 2008;1:357.

2. Chia VM, Li Y, Quraishi SM, et al. Effect modification of endocrine disruptors and testicular germ cell tumour risk by hormone-metabolizing genes. Int J Androl. 2010;33:588-596.

3. Ravenel JG, Gordon LL, Block MI, et al. Primary posterior mediastinal seminoma. AJR Am J Roentgenol. 2004;183:1835-1837.

4. Aparicio J, Maroto P, del Muro XG, et al. Risk-adapted treatment in clinical stage I testicular seminoma: the third Spanish germ cell cancer group study. J Clin Oncol. 2011;29:4677-4681.

5. Ruf CG, Linbecker M, Port M, et al. Predicting metastasized seminoma using gene expression. BJU Int. 2012;110:14-20.

6. Wudhikarn K, Colling CW, Robinson RA, et al. Solitary bony metastasis in seminoma. J Clin Oncol. 2013;31:259-261.

7. Crockford GP, Linger R, Hockley S, et al. Genome-wide linkage screen for testicular germ cell tumour susceptibility loci. Hum Mol Genet. 2006;15:443-451.

8. Jones DA, Ester EC, Leavitt D, et al. Adjuvant radiotherapy for synchronous bilateral testicular seminoma: a case report and a review of the pertinent literature. Case Rep Urol. 2013;2013:241073. 
9. Jiang X, Li D, Yang J, et al. Characterization of a novel human testisspecific gene: testis developmental related gene 1 (TDRG1). Tohoku J Exp Med. 2011;225:311-318.

10. Gan Y, Yang J, Wang Y, et al. In vitro study on shRNA-mediated reduction of testis developmental related gene 1 expression and its effects on the proliferation, invasion and apoptosis of NTERA-2 cells. Oncol Lett. 2015;10:61-66.

11. Zhang H, Bajraszewski N, Wu E, et al. PDGFRs are critical for PI3K/ Akt activation and negatively regulated by mTOR. J Clin Invest. 2007; 117:730-738.

12. Engelman JA. Targeting PI3K signalling in cancer: opportunities, challenges and limitations. Nat Rev Cancer. 2009;9:550-562.

13. Sheppard K, Kinross KM, Solomon B, et al. Targeting PI3 kinase/AKT/ mTOR signaling in cancer. Crit Rev Oncog. 2012;17:69-95.

14. Sun Y, Zhao S, Tian H, et al. Depletion of PI3K p85alpha induces cell cycle arrest and apoptosis in colorectal cancer cells. Oncol Rep. 2009; 22:1435-1441.

15. Liu N, Rowley BR, Bull CO, et al. BAY 80-6946 is a highly selective intravenous PI3K inhibitor with potent $\mathrm{p} 110 \alpha$ and $\mathrm{p} 110 \delta$ activities in tumor cell lines and xenograft models. Mol Cancer Ther. 2013;12:2319-2330.

16. Kitagishi Y, Kobayashi M, Kikuta K, et al. Roles of PI3K/AKT/GSK3/ mTOR pathway in cell signaling of mental illnesses. Depress Res Treat. 2012;2012:752563.

17. Manning BD, Cantley LC. AKT/PKB signaling: navigating downstream. Cell. 2007;129:1261-1274.

18. Lee TJ, Sartor O, Luftig RB, et al. Saposin C promotes survival and prevents apoptosis via PI3K/Akt-dependent pathway in prostate cancer cells. Mol Cancer. 2004;3:31.

19. Sekulić A, Hudson CC, Homme JL, et al. A direct linkage between the phosphoinositide 3-kinase-AKT signaling pathway and the mammalian target of rapamycin in mitogen-stimulated and transformed cells. Cancer Res. 2000;60:3504-3513.

20. Adhami VM, Syed DN, Khan N, et al. Dietary flavonoid fisetin: a novel dual inhibitor of PI3K/Akt and mTOR for prostate cancer management. Biochem Pharmacol. 2012;84:1277-1281.

21. Beevers CS, Li F, Liu L, et al. Curcumin inhibits the mammalian target of rapamycin-mediated signaling pathways in cancer cells. Int J Cancer. 2006;119:757-764.

22. Ouyang J, Shen YC, Yeh LK, et al. Pax6 overexpression suppresses cell proliferation and retards the cell cycle in corneal epithelial cells. Invest Ophthalmol Vis Sci. 2006;47:2397-2407.

23. Paterlini P, Suberville AM, Zindy F, et al. Cyclin A expression in human hematological malignancies: a new marker of cell proliferation. Cancer Res. 1993;53:235-238.

24. Lo YL, Lee HP, Tu WC. The use of a liposomal formulation incorporating an antimicrobial peptide from tilapia as a new adjuvant to epirubicin in human squamous cell carcinoma and pluripotent testicular embryonic carcinoma cells. Int J Mol Sci. 2015;16:22711-22734.

25. Ou CC, Hsu SC, Hsieh YH, et al. Downregulation of HER2 by RIG1 involves the PI3K/Akt pathway in ovarian cancer cells. Carcinogenesis. 2008;29:299-306.

26. Johnson SM, Gulhati P, Rampy BA, et al. Novel expression patterns of $\mathrm{PI} 3 \mathrm{~K} / \mathrm{Akt} / \mathrm{mTOR}$ signaling pathway components in colorectal cancer. J Am Coll Surg. 2010;210:767-778.

OncoTargets and Therapy

\section{Publish your work in this journal}

OncoTargets and Therapy is an international, peer-reviewed, open access journal focusing on the pathological basis of all cancers, potential targets for therapy and treatment protocols employed to improve the management of cancer patients. The journal also focuses on the impact of management programs and new therapeutic agents and protocols on
27. Xu Q, Ma J, Lei J, et al. $\alpha$-Mangostin suppresses the viability and epithelial-mesenchymal transition of pancreatic cancer cells by downregulating the PI3K/Akt pathway. Biomed Res Int. 2014;2014:546353.

28. Cheville JC, Rao S, Iczkowski KA, et al. Cytokeratin expression in seminoma of the human testis. Am J Clin Pathol. 2000;113:583-588.

29. Siegel C. Tumors and tumor like lesions of the testis: radiologicpathologic correlation. J Urol. 2003;169:1599.

30. Chetty C, Lakka SS, Bhoopathi P, et al. MMP-2 alters VEGF expression via alphaVbeta3 integrin-mediated PI3K/AKT signaling in A549 lung cancer cells. Int J Cancer. 2010;127:1081-1095.

31. Xie X, Tang B, Zhou J, et al. Inhibition of the PI3K/Akt pathway increases the chemosensitivity of gastric cancer to vincristine. Oncol Rep. 2013;30:773-782.

32. Nagoshi T, Matsui T, Aoyama T, et al. PI3K rescues the detrimental effects of chronic Akt activation in the heart during ischemia/reperfusion injury. J Clin Invest. 2005;115:2128-2138.

33. Maeno $\mathrm{Y}, \mathrm{Li} \mathrm{Q}$, Park K, et al. Inhibition of insulin signaling in endothelial cells by protein kinase $\mathrm{C}$-induced phosphorylation of p85 subunit of phosphatidylinositol 3-kinase (PI3K). J Biol Chem. 2012;287:4518-4530.

34. Cosentino C, Di Domenico M, Porcellini A, et al. p85 regulatory subunit of PI3K mediates cAMP-PKA and estrogen's biological effects on growth and survival. Oncogene. 2007;26:2095-2103.

35. Neal CL, Xu J, Li P, et al. Overexpression of $14-3-3 \zeta$ in cancer cells activates $\mathrm{PI} 3 \mathrm{~K}$ via binding the $\mathrm{p} 85$ regulatory subunit. Oncogene. 2012;31:897-906

36. Vivanco I, Sawyers CL. The phosphatidylinositol 3-kinase AKT pathway in human cancer. Nat Rev Cancer. 2002;2:489-501.

37. Zhang L, Huang J, Yang N, et al. Integrative genomic analysis of phosphatidylinositol 3'-kinase family identifies PIK3R3 as a potential therapeutic target in epithelial ovarian cancer. Clin Cancer Res. 2007;13:5314-5321.

38. De Santis G, Miotti S, Mazzi M, et al. E-cadherin directly contributes to PI3K/AKT activation by engaging the PI3K-p85 regulatory subunit to adherens junctions of ovarian carcinoma cells. Oncogene. 2009;28:1206-1217.

39. Campbell IG, Russell SE, Choong DY, et al. Mutation of the PIK3CA gene in ovarian and breast cancer. Cancer Res. 2004;64:7678-7681.

40. Miller TW, Rexer BN, Garrett JT, et al. Mutations in the phosphatidylinositol 3-kinase pathway: role in tumor progression and therapeutic implications in breast cancer. Breast Cancer Res. 2011;13:224.

41. Cheng SW, Fryer LG, Carling D, et al. Thr2446 is a novel mammalian target of rapamycin (mTOR) phosphorylation site regulated by nutrient status. J Biol Chem. 2004;279:15719-15722.

42. Soliman GA, Acosta-Jaquez HA, Dunlop EA, et al. mTOR Ser2481 autophosphorylation monitors mTORC-specific catalytic activity and clarifies rapamycin mechanism of action. $J$ Biol Chem. 2010;285:7866-7879.

43. Broek RV, Mohan S, Eytan DF, et al. The PI3K/Akt/mTOR axis in head and neck cancer: functions, aberrations, crosstalk, and therapies. Oral Dis. 2013;21(7):815-825.

44. Slomovitz BM, Coleman RL. The PI3K/AKT/mTOR pathway as a therapeutic target in endometrial cancer. Clin Cancer Res. 2012;18: 5856-5864.

patient perspectives such as quality of life, adherence and satisfaction. The manuscript management system is completely online and includes a very quick and fair peer-review system, which is all easy to use. Visit http://www.dovepress.com/testimonials.php to read real quotes from published authors. 